MATHEMATICS OF COMPUTATION

Volume 66, Number 218, April 1997, Pages 465-476

S 0025-5718(97)00837-5

\title{
A POSTERIORI ERROR ESTIMATE FOR THE MIXED FINITE ELEMENT METHOD
}

\author{
CARSTEN CARSTENSEN
}

\begin{abstract}
A computable error bound for mixed finite element methods is established in the model case of the Poisson-problem to control the error in the $\mathrm{H}(\operatorname{div}, \Omega) \times L^{2}(\Omega)$-norm. The reliable and efficient a posteriori error estimate applies, e.g., to Raviart-Thomas, Brezzi-Douglas-Marini, and Brezzi-DouglasFortin-Marini elements.
\end{abstract}

\section{MiXed Method For the Poisson problem}

Mixed finite element methods are well-established in the numerical treatment of partial differential equations as regards a priori error estimates to guarantee convergence $[\mathrm{BF}]$. In practical applications, a posteriori error control is at least of the same importance to guarantee a reliable approximation. Moreover, a posteriori error estimators indicate adaptive mesh-refinement criteria [EEHJ, V1] for an efficient computation.

In this paper we establish an efficient and reliable error estimator for the model example in the mixed finite element methods: Given $f \in L^{2}(\Omega)$, the Poisson problem consists in finding a function $u \in H_{0}^{1}(\Omega)$ that satisfies

$$
\operatorname{div}(A \nabla u)+f=0 \quad \text { in } \Omega .
$$

Here, $A \in L^{\infty}\left(\Omega ; \mathbb{R}^{2 \times 2}\right)$ is symmetric and uniformly elliptic, $\Omega$ is a convex bounded domain in the plane with polygonal boundary $\Gamma$. The Lebesgue and Sobolev spaces $L^{2}(\Omega)$ and $H_{0}^{1}(\Omega)$ are defined as usual (e.g., as in [H, LM]). We assume below that (1.1) is $H^{2}$-regular which, according to $\Omega$ being convex, means certain regularity on $A$ ( $A$ the unit matrix as for the Laplace equation is clearly sufficient).

The mixed formulation is given by splitting (1.1) into two equations where $u \in$ $H_{0}^{1}(\Omega)$ and $p \in L^{2}(\Omega)^{2}$ are unknown and have to satisfy

$$
\operatorname{div} p+f=0 \quad \text { and } \quad p=A \nabla u \quad \text { in } \Omega .
$$

It is well-known that (1.2) has a solution $(p, u) \in \mathrm{H}(\operatorname{div}, \Omega) \times L^{2}(\Omega)$, where, as usual, $\mathrm{H}(\operatorname{div}, \Omega):=\left\{q \in L^{2}(\Omega)^{2}: \operatorname{div} q \in L^{2}(\Omega)\right\}$ is endowed with the norm given by

$$
\|q\|_{\mathrm{H}(\operatorname{div}, \Omega)}^{2}:=\int_{\Omega}\left(|q|^{2}+|\operatorname{div} q|^{2}\right) d x \quad(q \in \mathrm{H}(\operatorname{div}, \Omega)) .
$$

Received by the editor September 12, 1995 and, in revised form, May 1, 1996.

1991 Mathematics Subject Classification. Primary 65N30, 65R20, 73C50.

Key words and phrases. Mixed finite element methods, a posteriori error estimates, adaptive algorithm.

(C)1997 American Mathematical Society 
The numerical approximation to $(u, p)$ consists in prescribing finite dimensional subspaces $L_{h}$ and $M_{h}$ of $L^{2}(\Omega)$ and $\mathrm{H}(\operatorname{div}, \Omega)$, respectively, and computing $\left(p_{h}, u_{h}\right) \in$ $M_{h} \times L_{h}$ that satisfies for all $\left(q_{h}, v_{h}\right) \in M_{h} \times L_{h}$

$$
\begin{aligned}
\int_{\Omega}\left(A^{-1} \cdot p_{h}\right) \cdot q_{h} d x+\int_{\Omega} u_{h} \cdot \operatorname{div} q_{h} d x & =0, \\
\int_{\Omega} v_{h} \cdot \operatorname{div} p_{h} d x & =-\int_{\Omega} v_{h} \cdot f d x .
\end{aligned}
$$

It is well-known that the discrete problem (1.3) has a unique solution if a discrete inf-sup-condition holds for the discrete spaces $M_{h}$ and $L_{h}[\mathrm{BF}]$ so we are interested in controlling the error

$$
\epsilon:=p-p_{h} \in \mathrm{H}(\operatorname{div}, \Omega) \text { and } \quad e:=u-u_{h} \in L^{2}(\Omega) .
$$

Moreover, if the discrete inf-sup-condition holds uniformly in $h$ we have a constant $c_{1}>0$ such that

$$
\|(\epsilon, e)\|_{\mathrm{H}(\operatorname{div}, \Omega) \times L^{2}(\Omega)} \leq c_{1} \cdot \inf _{\left(q_{h}, v_{h}\right) \in M_{h} \times L_{h}}\left\|\left(p-q_{h}, u-v_{h}\right)\right\|_{\mathrm{H}(\operatorname{div}, \Omega) \times L^{2}(\Omega)},
$$

i.e., the error is bounded from above and below by a constant times the bestapproximation error. We refer to $[\mathrm{BF}]$ for the setting, examples, proofs, and more details. The Raviart-Thomas, Brezzi-Douglas-Marini, and Brezzi-Douglas-FortinMarini elements are also described in $§ 3.1$.

\section{A posteriori ERror estimator}

In the mixed finite element method, we consider a regular triangulation $\mathcal{T}_{h}$ of $\Omega$ satisfying the angle condition (cf. $\S 4$ for explanations) and define, for each $T \in \mathcal{T}_{h}$, $h_{T}$ as the diameter of $T$, and, for any edge $E$ of $T$, let $J\left(p_{h} \cdot t\right)$ denote the jump of $p_{h} \cdot t$ across $E$ with $t$ being the tangential unit vector along $E ; h_{E}$ denotes the length of $E$. Then, define

$$
\begin{aligned}
\eta_{T}^{2}:= & \left\|f+\operatorname{div} p_{h}\right\|_{L^{2}(T)}^{2}+h_{T}^{2} \cdot\left\|\operatorname{curl}\left(A^{-1} p_{h}\right)\right\|_{L^{2}(T)}^{2} \\
& +h_{T}^{2} \cdot \min _{v_{h} \in L_{h}}\left\|A^{-1} p_{h}-\nabla_{h} v_{h}\right\|_{L^{2}(T)}^{2}+\left\|h_{E}^{1 / 2} J\left(A^{-1} p_{h} \cdot t\right)\right\|_{L^{2}(\partial T)}^{2}
\end{aligned}
$$

for any $T \in \mathcal{T}_{h}$ and consider the sum of all element contributions

$$
\eta_{h}:=\left(\sum_{T \in \mathcal{T}_{h}} \eta_{T}^{2}\right)^{1 / 2} .
$$

It is the aim of this paper to establish the following a posteriori error estimate.

Theorem 2.1. For the Raviart-Thomas, the Brezzi-Douglas-Marini, or the BrezziDouglas-Fortin-Marini elements there is a positive constant $c_{1}$ which only depends on $A, \Omega$, and on the shape of the elements and their polynomial degree $k$, such that

$$
\|(\epsilon, e)\|_{H(\operatorname{div}, \Omega) \times L^{2}(\Omega)} \leq c_{2} \cdot \eta_{h} .
$$

Moreover, the reverse inequality holds as well provided that on each $T \in \mathcal{T}_{h}$, $\left.A^{-1} p_{h}\right|_{T} \in \mathcal{P}_{\ell}$ and $\left.\nabla_{h} u_{h}\right|_{T} \in \mathcal{P}_{\ell} ; \mathcal{P}_{k}$ denotes the set of polynomials in two variables of total degree at most $k$. (Again, $A$ the unit matrix as for the Laplace equation is clearly sufficient.)

Theorem 2.2. For the Raviart-Thomas, the Brezzi-Douglas-Marini, or the BrezziDouglas-Fortin-Marini elements there is a positive constant $c_{3}$ which only depends 
on $A, \Omega$, and on the shape of the elements and the polynomial degrees $k$ and $\ell$, such that

$$
c_{3} \cdot \eta_{h} \leq\|(\epsilon, e)\|_{H(d i v, \Omega) \times L^{2}(\Omega)} .
$$

The proofs of Theorems 2.1 and 2.2 will be given in $\S \S 4-6$ under sharper but more technical assumptions while we first precede with some remarks in $\S 3$.

\section{REMARKS}

Some supplements are in order to comment on the results displayed in Theorems 2.1 and 2.2 .

3.1. Examples for mixed finite elements. The examples mentioned in Theorems 2.1 and 2.2 are briefly described for triangles $T \in \mathcal{T}_{h}$ by some $D_{k}(T) \subset \mathcal{C}(T)$ and $M_{k}(T) \subset \mathcal{C}(T)$ given in the following table where $k \geq 0$ and RT indicates entries for the Raviart-Thomas elements, BDM for the Brezzi-Douglas-Marini elements, and BDFM for the Brezzi-Douglas-Fortin-Marini elements.

\begin{tabular}{|c|cc|}
\hline Element & $M_{k}(T)$ & $D_{k}(T)$ \\
\hline RT & $\mathcal{P}_{k}^{2}+x \cdot \mathcal{P}_{k}$ & $\mathcal{P}_{k}$ \\
BDM & $\mathcal{P}_{k+1}^{2}$ & $\mathcal{P}_{k}$ \\
BDFM & $\left\{q \in \mathcal{P}_{k+1}^{2}:\left.(q \cdot n)\right|_{\partial T} \in \mathcal{R}_{k}(\partial T)\right\}$ & $\mathcal{P}_{k}$ \\
\hline
\end{tabular}

Here, $\mathcal{P}_{k}$ denotes polynomials of total degree at most $k$ and $\mathcal{R}_{k}(\partial T)$ denotes (not necessarily continuous) functions on $\partial T$ which equal a polynomial of degree at most $k$ on each edge of $T$. With the above sets $D_{k}(T)$ and $M_{k}(T)$ we define

$$
\begin{aligned}
L_{h} & :=\left\{v_{h} \in L^{2}(\Omega):\left.\forall T \in \mathcal{T}_{h} v_{h}\right|_{T} \in D_{k}(T)\right\}, \\
M_{h} & :=\left\{p_{h} \in \mathrm{H}(\operatorname{div}, \Omega):\left.\forall T \in \mathcal{T}_{h} v_{h}\right|_{T} \in M_{k}(T)\right\} .
\end{aligned}
$$

For more information, in particular about other elements in $\mathbb{R}^{n}$ and about practical implementations using multipliers, we refer to $[\mathrm{BF}]$.

3.2. Estimates in a weighted norm. The results in $\S \S 4-6$ give the following estimate with a different scaling in the equilibrium residual. Indeed, with $h: \Omega \rightarrow$ $(0, \infty)$ defined by $\left.h\right|_{T}=h_{T}$ on $T \in \mathcal{T}_{h}$ and by $\left.h\right|_{E}=h_{E}$ on $E \in \mathcal{E}_{h}$ there holds

$c_{4} \cdot \eta_{h, \kappa}\left(p_{h}, u_{h}\right) \leq\left\|A^{-1 / 2} \epsilon\right\|_{L^{2}(\Omega)}+\left\|h^{\kappa} \operatorname{div} \epsilon\right\|_{L^{2}(\Omega)}+\|e\|_{L^{2}(\Omega)} \leq c_{5} \cdot \eta_{h, \kappa}\left(p_{h}, u_{h}\right)$

where $0 \leq \kappa \leq 1$ and $\left(\mathcal{E}_{h}\right.$ denotes the set of edges in $\mathcal{T}_{h}$ and $\left.\Gamma_{h}:=\bigcup \mathcal{E}_{h}\right)$

$$
\begin{aligned}
& \eta_{h, \kappa}\left(p_{h}, u_{h}\right):=\left\|h^{\kappa} \cdot\left(f+\operatorname{div} p_{h}\right)\right\|_{L^{2}(\Omega)}+\left\|h \cdot \operatorname{curl}\left(A^{-1} p_{h}\right)\right\|_{L^{2}(\Omega)} \\
& \quad+\min _{v_{h} \in L_{h}}\left\|h \cdot\left(A^{-1} p_{h}-\nabla_{h} v_{h}\right)\right\|_{L^{2}(\Omega)}+\left\|h^{1 / 2} \cdot J\left(A^{-1} p_{h} \cdot t\right)\right\|_{L^{2}\left(\Gamma_{h}\right)} .
\end{aligned}
$$

3.3. Estimates for the stress variables. The results in $\S \S 4-6$ give the following estimate for the stress variable $p-p_{h}$, where $0 \leq \kappa \leq 1$,

$$
\begin{aligned}
c_{6} \cdot \eta_{h, \kappa}\left(p_{h}\right) \leq & \left\|A^{-1 / 2} \epsilon\right\|_{L^{2}(\Omega)}+\left\|h^{\kappa} \operatorname{div} \epsilon\right\|_{L^{2}(\Omega)} \leq c_{7} \cdot \eta_{h, \kappa}\left(p_{h}\right), \\
\eta_{h, \kappa}\left(p_{h}\right):= & \left\|h^{\kappa} \cdot\left(f+\operatorname{div} p_{h}\right)\right\|_{L^{2}(\Omega)}+\left\|h \cdot \operatorname{curl}\left(A^{-1} p_{h}\right)\right\|_{L^{2}(\Omega)} \\
& +\left\|h^{1 / 2} \cdot J\left(A^{-1} p_{h} \cdot t\right)\right\|_{L^{2}\left(\Gamma_{h}\right)} .
\end{aligned}
$$

We emphasize that this estimate holds also if (1.1) is not $H^{2}$-regular, so $\Omega$ may be an arbitrary bounded Lipschitz domain and $A_{i j} \in L^{\infty}(\Omega)$ is sufficient. 
3.4. On the term $A^{-1} p_{h}-\nabla_{h} v_{h}$. In the definition of $\eta_{T}$, we may replace

$$
h_{T}^{2} \cdot \min _{v_{h} \in L_{h}}\left\|A^{-1} p_{h}-\nabla_{h} v_{h}\right\|_{L^{2}(T)}^{2}
$$

by its upper bound

$$
h_{T}^{2} \cdot\left\|A^{-1} p_{h}-\nabla_{h} u_{h}\right\|_{L^{2}(T)}^{2}
$$

without losing reliability and efficiency. Indeed, we conclude from Lemma 6.3 in $\S 6$ that Theorem 2.2 remains valid for this modified (less sharp but possibly simpler) estimator.

3.5. Other estimates for the displacements. The preceding estimates for the stress variables and standard arguments in the theory of mixed finite element methods give a posteriori bounds for $\Pi_{L_{h}} e$ and $u-u_{h}^{*}$ where $u_{h}^{*}$ is the improved displacement field taking Lagrange multipliers in a practical implementation into account. Following the lines in $[\mathrm{BF}, \mathrm{p} .186]$ we can verify that

$$
\left\|\Pi_{L_{h}} u-u_{h}\right\|_{L^{2}(\Omega)} \leq c_{8} \cdot\left(\left\|h \cdot A^{-1 / 2} \epsilon\right\|_{L^{2}(\Omega)}+\|h \cdot \operatorname{div} \epsilon\|_{L^{2}(\Omega)}\right)
$$

which proves the a posteriori error estimate, $h_{\max }:=\|h\|_{L^{\infty}(\Omega)}$,

$$
\left\|\Pi_{L_{h}} u-u_{h}\right\|_{L^{2}(\Omega)} \leq c_{9} \cdot h_{\max } \cdot \eta_{h, 1}\left(p_{h}\right) .
$$

Furthermore, let $\mathcal{L}_{k}^{1, N C}:=\left\{v_{h} \in L^{2}(\Omega):\left.\forall T \in \mathcal{T}_{h} \forall \psi \in \mathcal{R}_{k}(\partial T) v_{h}\right|_{T} \in \mathcal{P}_{k} \wedge\right.$ $\left.\int_{\partial T} J\left(u_{h}\right) \cdot \psi d s=0\right\}$, let $u_{h}^{*} \in \mathcal{L}_{k}^{1, N C}$ denote the improved discrete displacement field defined in [BF, p.187] and let $\tilde{u}_{h}$ denote the $L^{2}(\Omega)$-best approximation to $u$ in $\mathcal{L}_{k}^{1, N C}$. Then, as shown in [BF, Eq. (3.13)],

$$
\left\|\tilde{u}_{h}-u_{h}^{*}\right\|_{L^{2}(\Omega)} \leq c_{10} \cdot\left(\|h \cdot \epsilon\|_{L^{2}(\Omega)}+\left\|\Pi_{L_{h}} e\right\|_{L^{2}(\Omega)}\right)
$$

which results in the a posteriori error estimate

$$
\left\|\tilde{u}_{h}-u_{h}^{*}\right\|_{L^{2}(\Omega)} \leq c_{11} \cdot h_{\max } \cdot \eta_{h, 1}\left(p_{h}\right) .
$$

3.6. Comments on the estimator by Braess and Verfürth. Braess and Verfürth established a posteriori error estimates for mixed methods in $[\mathrm{BV}]$ involving integration by parts in $\int_{\Omega} u_{h} \cdot \operatorname{div} q d x$ (which appears, e.g., in (1.3)). Since $u_{h}$ jumps across interelement boundaries those jumps count in their error indicator. Braess and Verfürth designed an error estimator working in mesh-dependent norms which is reliable and efficient in those norms but, somehow, is not efficient in the natural norm of $\mathrm{H}(\operatorname{div}, \Omega) \times L^{2}(\Omega)$, seemingly because the displacement variable is overestimated in their mesh-dependent norm. In this paper, we outlaw any such integration by parts (with one well-chosen exception, cf. (5.12) below) and so jumps of displacements cannot arise at all. Instead, we emphasize a Helmholtz decomposition and are led to the estimator $\eta_{h}$ which is reliable and efficient in the natural norm and avoids the saturation assumption that is important in [BV].

3.7. Comments on $A$. To estimate $e$ in the proof of Theorem 2.1, we need that (1.1) is $H^{2}$-regular (see $\S 4.2$ below for details). Since $\Omega$ is convex, the condition $A \in C^{1,0}(\bar{\Omega})$ is sufficient for that (see, e.g., [G, Thm 3.2.1.2] for a proof). Moreover, even some discontinuities are allowed, because we only need that the restriction $\left.u\right|_{T}$ of a solution $u$ to an element $T$ belongs to $H^{2}(T)$ (cf., (4.2) below). The following example proves that there exist problems (1.1) which are not $H^{2}$-regular but satisfy this assumption. 
Example 1. Let $A(x)=\rho(x) \cdot I_{2 \times 2}\left(I_{2 \times 2}\right.$ the $2 \times 2$-unit matrix $)$, and $\rho(x)>0$ is piecewise constant (with polygonal lines of discontinuities), the possible singularities of such transmission problems are understood and some of them lead to $H^{2}$-regular problems (see, e.g., [N, Sec. 2.4] and the references quoted therein). For example, consider a square $\Omega:=(0,1)^{2}$ and halve it along a diagonal $D:=\overline{T_{1}} \cap \overline{T_{2}}$ into two (open) congruent triangles $T_{1}$ and $T_{2}$. Let $\rho(x)=\rho_{j}$ for $x \in T_{j}, j=1,2$, for two positive constants $\rho_{1} \neq \rho_{2}$.

Then any $u \in H_{0}^{1}(\Omega)$ with $\operatorname{div}(A \nabla u) \in L^{2}(\Omega)$ satisfies $\left.u\right|_{T_{j}} \in H^{2}\left(T_{j}\right)$ for $j=1,2$, but (1.1) is not $H^{2}$-regular.

Proof. The natural interface conditions along $D$ show that $u \notin H^{2}(\Omega)$ (provided the normal derivatives (and hence their jump accross $D$ ) are non-zero which is generically the case). A careful study of the corner singularities tells us that $\left.u\right|_{T_{j}} \in$ $H^{2}\left(T_{j}\right)$ for $j=1,2$. (See, e.g., [N, Example 2.4] for a proof of that - there, it suffices to check that $D_{2}^{D}(\lambda)=0$ and $\lambda>0$ is possible only for $2 \geq \lambda$; cf., [N, page 102] for details and notation.)

In Theorem 2.2 we stated the condition that $A^{-1} p_{h}$ is a polynomial on each element (but may be discontinuous on interelement boundaries). In the examples of $\S 3.1,\left.p_{h}\right|_{T}$ is a polynomial so that $A^{-1}$ is required to be a polynomial too. The analysis in $\S 6$ shows that this restriction can be weakened. Actually, $A^{-1}$ has to be approximated by some polynomial $A_{T}^{-1}$ for which we precede as in the proof given below while some additional approximation error $\left\|A^{-1}-A_{T}^{-1}\right\|_{L^{\infty}(\Omega)}$ arises in the bounds.

3.8. Adaptive algorithms. As in many contributions to self-adapting meshrefinements (see, e.g., [EEHJ, V1, V2, V3] and the references quoted therein), based on an error estimator $\eta_{h}$ we get an algorithm for efficient mesh-design: For each mesh $\mathcal{T}_{h_{L}}$ with a Galerkin solution $\left(p_{h_{L}}, u_{h_{L}}\right)$ and local error estimators $\eta_{T}$, we refine $T \in \mathcal{T}_{h_{L}}$ (e.g., by halving its largest side) if (for example)

$$
\eta_{T} \geq 0.5 \cdot \max _{T^{\prime} \in \mathcal{T}_{h_{L}}} \eta_{T^{\prime}}
$$

Then, further refinements to avoid hanging nodes lead to a new mesh $\mathcal{T}_{h_{L+1}}$ from which we start again.

\section{Preliminaries}

Theorem 2.1 holds under the following weaker assumptions on $\mathcal{T}_{h}, A$, on $L_{h} \subset$ $L^{2}(\Omega)$ and $M_{h} \subset \mathrm{H}(\operatorname{div}, \Omega)$. We emphasize that the Raviart-Thomas, BrezziDouglas-Marini, and Brezzi-Douglas-Fortin-Marini elements satisfy all the assumptions in this section.

4.1. Assumptions on $\Omega$. The bounded Lipschitz domain $\Omega$ is assumed to be convex with a polygonal boundary. Depending only on $\Omega$ we have a constant $c_{12}>0$ such that, for all $v \in H^{1}(\Omega)$ with integral mean $v_{0}$, Poincaré's inequality reads

$$
\left\|v-v_{0}\right\|_{2, \Omega} \leq c_{12} \cdot\|\nabla v\|_{2, \Omega}
$$


4.2. Assumptions on $A$. We assume that $A \in L^{\infty}\left(\Omega ; \mathbb{R}_{\text {sym }}^{2 \times 2}\right)$ is uniformly elliptic, i.e., $A(x)$ is a symmetric and positive definite $2 \times 2$-matrix, with eigenvalues $\lambda_{j}(x) \in$ $\mathbb{R}$ satisfying $0<c_{A} \leq \lambda_{1}(x), \lambda_{2}(x) \leq C_{A}$ for almost all $x \in \Omega$. Then, by the LaxMilgram lemma, the operator

$$
-\operatorname{div}(A \nabla \cdot): H_{0}^{1}(\Omega) \rightarrow H^{-1}(\Omega)
$$

is invertible and the norm of the inverse is bounded by a constant $c_{13}>0$ depending on $c_{A}$ and $c_{12}$. Moreover, since $\Omega$ is convex, $A \in \mathcal{C}^{1,0}(\bar{\Omega})$ implies that

$$
-\operatorname{div}(A \nabla \cdot): H_{0}^{1}(\Omega) \cap H^{2}(\Omega) \rightarrow L^{2}(\Omega)
$$

is invertible $[\mathrm{G}]$ and there is a constant $c_{14}>0$ such that

$\|v\|_{2,2, U \mathcal{T}_{h}} \leq c_{14} \cdot\|\operatorname{div}(A \nabla v)\|_{2, \Omega} \quad\left(v \in H_{0}^{1}(\Omega)\right.$ such that $\left.\operatorname{div}(A \nabla v) \in L^{2}(\Omega)\right)$.

We emphasize that we only need an estimate on $\|v\|_{2,2, T}$ for each $T \in \mathcal{T}_{h}$, i.e., the assumption on $A$ could be weakened in the sense that only (4.2) is required (cf., Example 1 where $A$ is piecewise constant and satisfies (4.2) but (1.1) is not $H^{2}$-regular).

Finally, we need that $A$ is elementwise smooth assuming that there exists a constant $c_{15}>0$ such that

$$
\max _{i, j, k=1,2}\left\|\left(\nabla_{h} A\right)_{i j k}\right\|_{\infty, \cup \mathcal{T}_{h}} \leq c_{15}
$$

4.3. Assumptions on $\mathcal{T}_{h}$. The triangulation $\mathcal{T}_{h}$ is assumed to be regular in the sense of $[\mathrm{C}]$ and satisfies the angle condition which means that there is a constant $c_{16}>0$ such that for all $T \in \mathcal{T}_{h}$

$$
c_{16}^{-1} \cdot h_{T}^{2} \leq|T| \leq c_{16} \cdot h_{T}^{2}
$$

where $|T|$ is the area of $T$. We define $S^{0}\left(\mathcal{T}_{h}\right) \subset L^{2}(\Omega)$ as the piecewise constant and $S^{1}\left(\mathcal{T}_{h}\right) \subset H^{1}(\Omega)$ or $S_{0}^{1}\left(\mathcal{T}_{h}\right) \subset H_{0}^{1}(\Omega)$ as continuous and piecewise affine functions; piecewise is understood with respect to $\mathcal{T}_{h}$. We consider Clement's interpolation operator $[\mathrm{Cl}] r_{h}: H^{1}(\Omega) \rightarrow S^{1}\left(\mathcal{T}_{h}\right)$ which satisfies

$$
\begin{aligned}
& \left\|v-r_{h} v\right\|_{2, T} \leq c_{17} \cdot h_{T} \cdot\|v\|_{1,2, \omega_{T}} \\
& \left\|v-r_{h} v\right\|_{2, E} \leq c_{18} \cdot h_{E}^{1 / 2} \cdot\|v\|_{1,2, \omega_{E}}
\end{aligned} \quad\left(v \in H_{0}^{1}(\Omega)\right)
$$

for each $T \in \mathcal{T}_{h}$ and $E \in \mathcal{E}_{h}, \mathcal{E}_{h}$ being the set of element sides in $\mathcal{T}_{h}$. Here and below, $\|\cdot\|_{p, \omega}$ denotes the norm in $L^{p}(\omega)$ for $\omega \subset \Omega$ as well as for some edge $\omega=E$ while $\|\cdot\|_{m, p, \omega}$ and $|\cdot|_{m, p, \omega}$ denote norm and semi-norm in $W^{m, p}(\omega)$, respectively; in particular, we will occasionally write $\|\cdot\|_{2}$ instead of $\|\cdot\|_{2, \Omega}$ and $H^{m}(\omega)$ instead of $W^{m, 2}(\omega)$. With $T \in \mathcal{T}_{h}$ and $E \in \mathcal{E}_{h}$ we associate neighbourhoods $\omega_{T}$ and $\omega_{E}$

$$
\omega_{T}:=\bigcup\left\{T^{\prime} \in \mathcal{T}_{h}: \bar{T} \cap \overline{T^{\prime}} \neq \emptyset\right\} \quad \text { and } \quad \omega_{E}:=\bigcup\left\{T \in \mathcal{T}_{h}: E \subset \bar{T}\right\} .
$$

Then, the positive constants $c_{17}$ and $c_{18}$ only depend on $c_{16}$. Moreover, let $c_{19}$ be the maximal number of elements in $\omega_{T}$ which is $h$-independently bounded by the angle condition (depending on $c_{16}$ ). For all $E \in \mathcal{E}_{h}$ we fix one direction of a unit normal on $E$ pointing in the outside of $\Omega$ in case that $E \subset \Gamma$. With $\Gamma_{h}:=\bigcup \mathcal{E}_{h}$ we define $J: H^{1}\left(\bigcup \mathcal{T}_{h}\right) \rightarrow L^{2}\left(\Gamma_{h}\right)$, for $E \subset \Gamma_{h}$ and $v \in H^{1}\left(\bigcup \mathcal{T}_{h}\right)$ by

$$
\left.J(v)\right|_{E}:=\left.\left(\left.v\right|_{T_{+}}\right)\right|_{E}-\left.\left(\left.v\right|_{T_{-}}\right)\right|_{E} \quad \text { if } \quad E=\overline{T_{+}} \cap \overline{T_{-}} \quad\left(E \in \mathcal{E}_{h} ; T_{+}, T_{-} \in \mathcal{T}_{h}\right)
$$


and $n_{E}$ points from $T_{+}$into its neighbour element $T_{-}$; while

$$
\left.J(v)\right|_{E}:=\left.\left(\left.v\right|_{T}\right)\right|_{E} \quad \text { if } \quad E=\bar{T} \cap \Gamma \quad\left(E \in \mathcal{E}_{h} ; T \in \mathcal{T}_{h}\right) .
$$

We define $W^{m, p}\left(\bigcup \mathcal{T}_{h}\right):=\left\{v \in L^{p}(\Omega):\left.\forall T \in \mathcal{T}_{h} v\right|_{T} \in W^{m, p}(T)\right\}$ and consider local versions of the differential operators div, $\nabla$, curl (understood in the distributional sense, i.e., in $\left.\mathcal{D}^{\prime}(\Omega)\right)$, namely, $\operatorname{div}_{h}, \operatorname{curl}_{h}: W^{1,2}\left(\bigcup \mathcal{T}_{h}\right)^{2} \rightarrow L^{2}(\Omega)$ and $\nabla_{h}: W^{1,2}\left(\bigcup \mathcal{T}_{h}\right) \rightarrow L^{2}(\Omega)$ defined such that, e.g.,

$$
\left.\operatorname{div}_{h} v\right|_{T}:=\operatorname{div}\left(\left.v\right|_{T}\right) \quad \text { in } \mathcal{D}^{\prime}(T) \quad\left(T \in \mathcal{T}_{h}\right)
$$

If there is no risk of confusion the local meshsize $h$ is defined on both $\Omega$ and $\Gamma_{h}:=\bigcup \mathcal{E}_{h}$ by $\left.h\right|_{T}:=h_{T}$ for $T \in \mathcal{T}_{h}$ and $\left.h\right|_{E}:=h_{E}$ for $E \in \mathcal{E}_{h}$, respectively.

4.4. Assumptions on $L_{h}$. We assume that $L_{h} \subset H^{1}\left(\bigcup \mathcal{T}_{h}\right)$ such that the $L^{2}(\Omega)-$ orthogonal projection $\Pi_{L_{h}}: L^{2}(\Omega) \rightarrow L_{h}$ satisfies

$$
\left\|v-\Pi_{L_{h}} v\right\|_{2, \Omega} \leq c_{4} \cdot\left\|h \cdot \nabla_{h} v\right\|_{2, \Omega} \quad\left(v \in H^{1}\left(\bigcup \mathcal{T}_{h}\right)\right)
$$

For example, if $S^{0}\left(\mathcal{T}_{h}\right) \subset L_{h}$, the Poincaré inequality (4.6) is satisfied with a positive constant $c_{20}$ which only depends on the shape of the elements.

Furthermore, for the lower bound (Theorem 2.2) we assume $\left.\left(\nabla_{h} u_{h}\right)\right|_{T} \in \mathcal{P}_{\ell}^{2}$ for all $T \in \mathcal{T}_{h}$.

4.5. Assumptions on $M_{h}$. We assume that

$$
S^{0}\left(\mathcal{T}_{h}\right)^{2} \cap \mathrm{H}(\operatorname{div}, \Omega) \subset M_{h} \subset H^{1}\left(\bigcup \mathcal{T}_{h}\right) \cap \mathrm{H}(\operatorname{div}, \Omega) \quad \text { and } \quad \operatorname{div} M_{h}=L_{h} .
$$

Furthermore, in Theorem 2.2, we assume $\left.\left(A^{-1} p_{h}\right)\right|_{T} \in \mathcal{P}_{\ell}^{2}$ for all $T \in \mathcal{T}_{h}$.

4.6. Assumptions on an interpolation operator $\Pi_{h}$. We assume that there exists an operator $\Pi_{h}: W \rightarrow M_{h}$ where $W=\mathrm{H}(\operatorname{div}, \Omega) \cap L^{s}(\Omega)^{2}$ for some $s>2$ as, e.g., in [BF, §III.3], such that the following diagram commutes

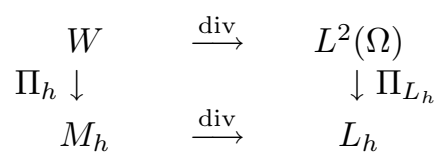

where $\Pi_{L_{h}}$ is the $L^{2}(\Omega)$-orthogonal projection. Let Id denote identity and let $\perp$ denote $L^{2}(\Omega)$-orthogonality. Then, the commuting diagram property in (4.8) reads

$$
\operatorname{div}\left(\operatorname{Id}-\Pi_{h}\right) W \perp L_{h}
$$

Further, we assume that the interpolant satisfies a local error estimate (note that $\left.H^{1}\left(\bigcup \mathcal{T}_{h}\right) \cap \mathrm{H}(\operatorname{div}, \Omega) \subset W\right)$

$$
\left\|h^{-1} \cdot\left(\operatorname{Id}-\Pi_{h}\right) q\right\|_{2} \leq c_{21} \cdot|q|_{1,2, \cup \mathcal{T}_{h}} \quad\left(q \in H^{1}\left(\bigcup \mathcal{T}_{h}\right) \cap \mathrm{H}(\operatorname{div}, \Omega)\right) .
$$

Finally, we assume that $\Pi_{h}$ approximates the normal components on element edges such that we have, for any $E \in \mathcal{E}_{h}$, for any $v_{h} \in L_{h}$, and for all $q \in W$,

$$
\int_{T} v_{h} \cdot\left(\mathrm{Id}-\Pi_{h}\right) q \cdot n_{E} d x=0 .
$$

We refer to $[\mathrm{BF}]$ for proofs, further explanations and explicit definitions of $\Pi_{h}$ in the examples under consideration. 


\section{Proof of Theorem 2.1}

Theorem 2.1 is a direct consequence of the following two lemmas and the fact that $-\operatorname{div} \epsilon=f+\operatorname{div} p_{h}$. We recall that the local meshsize $h$ is defined on $\Omega \backslash \Gamma_{h}$ by $\left.h\right|_{T}:=h_{T}$ for $T \in \mathcal{T}_{h}$ and on $\Gamma_{h}:=\bigcup \mathcal{E}_{h}$ by $\left.h\right|_{E}:=h_{E}$ for $E \in \mathcal{E}_{h}$.

Lemma 5.1. For $c_{22}:=\max \left\{\sqrt{8} c_{12} \cdot c_{18} \cdot C_{A}, \sqrt{2} c_{12} \cdot c_{17} \cdot c_{19} \cdot C_{A}, c_{20} \cdot c_{A}^{-1}\right\}$ we have

$$
\begin{aligned}
\left\|A^{-1 / 2} \epsilon\right\|_{2, \Omega} \leq & c_{22} \cdot\left(\left\|h \cdot \operatorname{curl}_{h}\left(A^{-1} p_{h}\right)\right\|_{2, \Omega}^{2}\right. \\
& \left.+\left\|h \cdot\left(f+\operatorname{div} p_{h}\right)\right\|_{2, \Omega}^{2}+\left\|h^{1 / 2} \cdot J\left(A^{-1 / 2} p_{h} \cdot t\right)\right\|_{2, \Gamma_{h}}^{2}\right)^{1 / 2} .
\end{aligned}
$$

Proof. We consider a Helmholtz decomposition of $A^{-1} p_{h}$ fixing $\alpha \in H_{0}^{1}(\Omega)$ with

$$
\operatorname{div}(A \nabla \alpha)=\operatorname{div} p_{h} \quad \text { in } \mathcal{D}^{\prime}(\Omega) .
$$

Then, there is some $\beta \in H^{1}(\Omega)$ satisfying $\int_{\Omega} \beta d x=0, \operatorname{Curl} \beta \perp \nabla H_{0}^{1}(\Omega)$, and

$$
p_{h}=A \nabla \alpha+\operatorname{Curl} \beta
$$

$\left(\perp\right.$ denotes $L^{2}(\Omega)$-orthogonality). From (1.2) and (5.2) we obtain

$$
\epsilon=A \nabla z-\operatorname{Curl} \beta \quad \text { with } \quad z:=u-\alpha \in H_{0}^{1}(\Omega)
$$

and hence the error decomposition

$$
\int_{\Omega}\left(A^{-1} \epsilon\right) \cdot \epsilon d x=\int_{\Omega}(A \nabla z) \cdot \nabla z d x+\int_{\Omega}\left(A^{-1} \operatorname{Curl} \beta\right) \cdot \operatorname{Curl} \beta d x .
$$

To estimate the first contribution of the right-hand side in (5.4) we integrate by parts and utilize $\operatorname{div} \epsilon \perp L_{h}$ (which follows from (1.2) and (1.3)). With (4.6), this leads to

$$
\begin{aligned}
& \int_{\Omega}(A \nabla z) \cdot \nabla z d x=\int_{\Omega} \nabla z \cdot \epsilon d x=-\int_{\Omega} z \cdot \operatorname{div} \epsilon d x \\
= & -\int_{\Omega}\left(z-\Pi_{L_{h}} z\right) \cdot \operatorname{div} \epsilon d x \leq c_{20} \cdot c_{A}^{-1} \cdot\|h \cdot \operatorname{div} \epsilon\|_{2} \cdot\left\|A^{1 / 2} \nabla z\right\|_{2} .
\end{aligned}
$$

To estimate the second contribution to the right-hand side of (5.4) we define $\beta_{h}:=$ $r_{h} \beta \in S^{1}\left(\mathcal{T}_{h}\right) \subset H^{1}(\Omega)$ utilizing Clement's operator $r_{h}$. Note that $\operatorname{Curl}_{h} \beta_{h}=$ $\operatorname{Curl} \beta_{h} \in S^{0}\left(\mathcal{T}_{h}\right)^{2} \subset L^{\infty}(\Omega)^{2}$ and $\operatorname{Curl} \beta_{h} \perp \nabla H_{0}^{1}(\Omega)$, whence div $\operatorname{Curl} \beta_{h}=0$, and

$$
\operatorname{Curl} \beta_{h} \in \mathrm{H}(\operatorname{div}, \Omega) \cap L^{\infty}(\Omega)^{2} \cap M_{h}
$$

according to (4.7). Therefore, (5.3) and (1.2)-(1.3) show

$$
\begin{gathered}
\int_{\Omega}\left(A^{-1} \operatorname{Curl} \beta\right) \cdot \operatorname{Curl} \beta_{h} d x=-\int_{\Omega}\left(A^{-1} \epsilon\right) \cdot \operatorname{Curl} \beta_{h} d x \\
=\int_{\Omega} e \cdot \operatorname{div} \operatorname{Curl} \beta_{h} d x=0 .
\end{gathered}
$$

From the integration by parts formula

$$
\int_{\omega}\left(\phi \cdot \frac{\partial \psi}{\partial x_{j}}+\psi \cdot \frac{\partial \phi}{\partial x_{j}}\right) d x=\int_{\partial \omega} \psi \cdot \phi \cdot n_{j} d s
$$

(say, for $\phi, \psi \in H^{1}(\omega), j=1,2, n=\left(n_{1}, n_{2}\right)^{T} \in \mathbb{R}^{2}$ the exterior unit-normal to the Lipschitz boundary $\partial \omega$ ) we gain

$$
\int_{\omega}(\psi \cdot \operatorname{Curl} \phi+\phi \cdot \operatorname{curl} \psi) d x=\int_{\partial \omega} \phi \cdot(\psi \cdot t) d s \quad\left(\phi, \psi_{1} \psi_{2} \in H^{1}(\omega)\right)
$$


where $t$ is tangential on $\partial \omega: t_{1}=-n_{2}, t_{2}=n_{1}$, and where we differentiate between curls involved as

$$
\operatorname{curl} \psi=\frac{\partial \psi_{2}}{\partial x_{1}}-\frac{\partial \psi_{1}}{\partial x_{2}} \quad \text { and } \quad \operatorname{Curl} \phi=\left(-\frac{\partial \phi}{\partial x_{2}}, \frac{\partial \phi}{\partial x_{1}}\right)^{T} .
$$

Utilizing (5.6), (5.2), and (5.7) we infer $\left(\right.$ recall $\left.\Gamma_{h}=\bigcup \mathcal{E}_{h}\right)$

$$
\begin{aligned}
\int_{\Omega}\left(A^{-1} \operatorname{Curl} \beta\right) \cdot \operatorname{Curl} \beta d x=\int_{\Omega} A^{-1} p_{h} \cdot \operatorname{Curl}\left(\beta-\beta_{h}\right) d x \\
\quad=-\int_{\Omega}\left(\beta-\beta_{h}\right) \cdot \operatorname{curl}_{h}\left(A^{-1} p_{h}\right) d x+\int_{\Gamma_{h}} J\left(A^{-1} p_{h} \cdot t\right) \cdot\left(\beta-\beta_{h}\right) d s .
\end{aligned}
$$

According to (4.5), and since the number of elements in $\omega_{T}$ is bounded by $c_{19}$,

$$
\begin{gathered}
\int_{\Omega}\left(\beta-\beta_{h}\right) \cdot \operatorname{curl}_{h}\left(A^{-1} p_{h}\right) d x \leq c_{17} \cdot c_{19} \cdot\left\|h \cdot \operatorname{curl}_{h}\left(A^{-1} p_{h}\right)\right\|_{2} \cdot\|\beta\|_{1,2, \Omega}, \\
\int_{\Gamma_{h}} J\left(A^{-1} p_{h} \cdot t\right) \cdot\left(\beta-\beta_{h}\right) d s \leq 2 c_{18} \cdot\left\|h^{1 / 2} \cdot J\left(A^{-1} p_{h} \cdot t\right)\right\|_{2, \Gamma_{h}} \cdot\|\beta\|_{1,2, \Omega} .
\end{gathered}
$$

With Poincaré's inequality (4.1) and ellipticity of $A$ we deduce

$$
\|\beta\|_{1,2, \Omega} \leq c_{12} \cdot\|\nabla \beta\|_{2, \Omega}=c_{12} \cdot\|\operatorname{Curl} \beta\|_{2, \Omega} \leq c_{12} \cdot C_{A} \cdot\left\|A^{-1 / 2} \operatorname{Curl} \beta\right\|_{2, \Omega} .
$$

The above estimates verify

$$
\begin{aligned}
& \int_{\Omega}\left(A^{-1} \operatorname{Curl} \beta\right) \cdot \operatorname{Curl} \beta d x \leq c_{23} \cdot\left\|A^{-1 / 2} \operatorname{Curl} \beta\right\|_{2, \Omega} \\
& \cdot\left(\left\|h^{1 / 2} \cdot J\left(A^{-1} p_{h} \cdot t\right)\right\|_{2, \Gamma_{h}}+\left\|h \cdot \operatorname{curl}_{h}\left(A^{-1} p_{h}\right)\right\|_{2}\right)
\end{aligned}
$$

where $c_{23}:=c_{12} \cdot C_{A} \cdot \max \left\{2 c_{18}, c_{17} \cdot c_{19}\right\}$. Together with (5.4) and (5.5) this establishes

$$
\begin{aligned}
\left\|A^{-1 / 2} \epsilon\right\|_{2}^{2} \leq & \left(c_{20}^{2} \cdot c_{A}^{-2} \cdot\|h \cdot \operatorname{div} \epsilon\|_{2}^{2}+2 c_{23}^{2} \cdot\left\|h^{1 / 2} \cdot J\left(A^{-1} p_{h} \cdot t\right)\right\|_{2, \Gamma_{h}}^{2}\right. \\
& \left.+2 c_{23}^{2} \cdot\left\|h \cdot \operatorname{curl}_{h}\left(A^{-1} p_{h}\right)\right\|_{2}^{2}\right)^{1 / 2} \cdot\left\|A^{-1 / 2} \epsilon\right\|_{2}
\end{aligned}
$$

and concludes the proof.

Lemma 5.2. For $c_{24}:=\left(c_{20}^{2} \cdot c_{A}^{-2}+c_{14}^{2} \cdot c_{21}^{2} \cdot\left(C_{A}^{2}+4 c_{15}^{2}\right)\right)^{1 / 2}$ we have

$$
\|e\|_{2, \Omega} \leq c_{24} \cdot\left(\left\|h \cdot\left(f+\operatorname{div} p_{h}\right)\right\|_{2, \Omega}^{2}+\min _{v_{h} \in L_{h}}\left\|h \cdot\left(A^{-1} p_{h}-\nabla_{h} v_{h}\right)\right\|_{2, \Omega}^{2}\right)^{1 / 2} .
$$

Proof. There exists exactly one $\eta \in H_{0}^{1}(\Omega)$ with $\operatorname{div}(A \nabla \eta)=e$. According to (4.2), we have $\eta \in H_{0}^{1}(\Omega) \cap H^{2}\left(\bigcup \mathcal{T}_{h}\right)$ and

$$
\max \left\{c_{14}^{-1} \cdot\|\eta\|_{2,2, \cup \mathcal{T}_{h}}, c_{13}^{-1} \cdot\|\eta\|_{1,2, \Omega}, c_{A} \cdot|\eta|_{1,2, \Omega}\right\} \leq\|e\|_{2, \Omega} .
$$


By construction of $\eta$, integration by parts and with (1.2), (1.3) and (4.9) we infer, for any $v_{h} \in L_{h}$,

$$
\begin{aligned}
\|e\|_{2}^{2} & =\int_{\Omega}\left(u-u_{h}\right) \cdot \operatorname{div}(A \nabla \eta) d x \\
& =-\int_{\Omega} p \cdot \nabla \eta d x-\int_{\Omega} u_{h} \cdot \operatorname{div} \Pi_{h}(A \nabla \eta) d x \\
& =-\int_{\Omega} \epsilon \cdot \nabla \eta d x-\int_{\Omega}\left(A^{-1} p_{h}\right) \cdot\left(\mathrm{Id}-\Pi_{h}\right)(A \nabla \eta) d x \\
& =\int_{\Omega} \eta \cdot \operatorname{div} \epsilon d x+\int_{\Omega}\left(\nabla_{h} v_{h}-A^{-1} p_{h}\right) \cdot\left(\operatorname{Id}-\Pi_{h}\right)(A \nabla \eta) d x \\
& -\int_{\Omega} \nabla_{h} v_{h} \cdot\left(\operatorname{Id}-\Pi_{h}\right)(A \nabla \eta) d x .
\end{aligned}
$$

Letting $\eta_{h}:=\Pi_{L_{h}} \eta$ we get from (1.2), (1.3), and (4.6) that

$$
\int_{\Omega} \eta \cdot \operatorname{div} \epsilon d x=\int_{\Omega}\left(\eta-\eta_{h}\right) \cdot \operatorname{div} \epsilon d x \leq c_{20} \cdot\left\|\nabla_{h} \eta\right\|_{2, \Omega} \cdot\|h \cdot \operatorname{div} \epsilon\|_{2, \Omega} .
$$

The second term on the right-hand side of (5.9) is

$$
\begin{aligned}
& \int_{\Omega}\left(\nabla_{h} v_{h}-A^{-1} p_{h}\right) \cdot\left(\mathrm{Id}-\Pi_{h}\right)(A \nabla \eta) d x \\
& \quad \leq\left\|h \cdot\left(\nabla_{h} v_{h}-A^{-1} p_{h}\right)\right\|_{2} \cdot\left\|h^{-1} \cdot\left(\mathrm{Id}-\Pi_{h}\right)(A \nabla \eta)\right\|_{2} .
\end{aligned}
$$

According to (4.10) and letting $c_{25}:=c_{21} \cdot\left(C_{A}^{2}+4 c_{15}^{2}\right)^{1 / 2}$ we obtain

$$
\left\|h^{-1} \cdot\left(\mathrm{Id}-\Pi_{h}\right)(A \nabla \eta)\right\|_{2, \Omega} \leq c_{21} \cdot|A \nabla \eta|_{1,2, \cup \mathcal{T}_{h}} \leq c_{25} \cdot\|\eta\|_{2,2, \cup \mathcal{T}_{h}}
$$

and conclude

$$
\begin{aligned}
& \int_{\Omega}\left(\nabla_{h} v_{h}-A^{-1} p_{h}\right) \cdot\left(\operatorname{Id}-\Pi_{h}\right)(A \nabla \eta) d x \\
& \quad \leq c_{25} \cdot\left\|h \cdot\left(\nabla_{h} v_{h}-A^{-1} p_{h}\right)\right\|_{2} \cdot\|\eta\|_{2,2, \cup \mathcal{T}_{h}} .
\end{aligned}
$$

The last term in (5.9) vanishes because the integral on $\Gamma_{h}$ in the integration by parts is zero by (4.11) and so

$$
\int_{\Omega} \nabla_{h} v_{h} \cdot\left(\operatorname{Id}-\Pi_{h}\right)(A \nabla \eta) d x=\int_{\Omega} v_{h} \cdot \operatorname{div}\left(\operatorname{Id}-\Pi_{h}\right)(A \nabla \eta) d x=0
$$

because of (4.9). Putting (5.9)-(5.12) together with (5.8) we have

$$
\|e\|_{2}^{2} \leq\|e\|_{2} \cdot\left(c_{20} \cdot c_{A}^{-1} \cdot\|h \cdot \operatorname{div} \epsilon\|_{2}+c_{14} \cdot c_{25} \cdot\left\|h \cdot\left(\nabla_{h} v_{h}-A^{-1} p_{h}\right)\right\|_{2}\right)
$$

and conclude the proof with Cauchy's inequality.

\section{Proof of Theorem 2.2}

As indicated by the additional hypothesis $\left.A^{-1} p_{h}\right|_{T} \in \mathcal{P}_{\ell}$ and $\left.\nabla_{h} u_{h}\right|_{T} \in \mathcal{P}_{\ell}$, the lower bound is proved by inverse inequalities - a technique already elaborated in [V1, V2, V3]. The setting is simple: various weighted norms on polynomials on the reference element are equivalent and that by transforming backwards and forwards the equivalence constants of the current element only depend further on the change of the shape (i.e. on $c_{16}$ ) and the scaling (i.e. on $h_{T}$ ) during these transformations.

The proof of Theorem 2.2 is divided into Lemmas 6.1-6.3 where the positive constants $c_{26}, \ldots, c_{36}$ arising below only depend on the shape of the elements, their maximal polynomial degree, and on $\ell$. 
Lemma 6.1. For each $T \in \mathcal{T}_{h}$ and with $\beta$ as defined in (5.2),

$$
h_{T} \cdot\left\|\operatorname{curl}\left(A^{-1} p_{h}\right)\right\|_{2, T} \leq c_{26} \cdot\left\|A^{-1 / 2} \operatorname{Curl} \beta\right\|_{2, T} .
$$

Proof. Fixing $\psi_{T} \in \mathcal{P}_{3}$ with $0 \leq \psi_{T} \leq 1=\max \psi$ and zero boundary values on $T$ we learn (e.g., from [V1, Lemma 1.3], or [V2, Lemma 4.1], or [V3, Lemma 5.1])

$$
c_{27} \cdot\left\|\operatorname{curl}\left(A^{-1} p_{h}\right)\right\|_{2, T}^{2} \leq\left\|\psi_{T}^{1 / 2} \cdot \operatorname{curl}\left(A^{-1} p_{h}\right)\right\|_{2, T}^{2} \cdot
$$

Integration by parts, $\operatorname{curl}_{h}\left(A^{-1} p_{h}\right)=-\operatorname{curl}_{h}\left(A^{-1} \epsilon\right)$, and (5.3) verify

$$
\begin{aligned}
\left\|\psi_{T}^{1 / 2} \cdot \operatorname{curl}\left(A^{-1} p_{h}\right)\right\|_{2, T}^{2} & =\int_{T}\left(A^{-1} \epsilon\right) \cdot \operatorname{Curl}\left(\psi_{T} \cdot \operatorname{curl}\left(A^{-1} p_{h}\right)\right) d x \\
& =-\int_{T}\left(A^{-1} \operatorname{Curl} \beta\right) \cdot \operatorname{Curl}\left(\psi_{T} \cdot \operatorname{curl}\left(A^{-1} p_{h}\right)\right) d x
\end{aligned}
$$

Since $\psi_{T} \cdot \operatorname{curl}\left(A^{-1} p_{h}\right) \in \mathcal{P}_{\ell+2}$ with zero boundary values on $T$ we have

$$
\left|\psi_{T} \cdot \operatorname{curl}\left(A^{-1} p_{h}\right)\right|_{1,2, T} \leq c_{28} \cdot h_{T}^{-1} \cdot\left\|\psi_{T} \cdot \operatorname{curl}\left(A^{-1} p_{h}\right)\right\|_{2, T}
$$

(as, e.g., in [V2, Lemma 4.1] or [V3, Lemma 5.1]). Finally, Cauchy's inequality, (5.4), and (6.2)-(6.4) prove the lemma.

Lemma 6.2. For each $E \in \mathcal{E}_{h}$,

$$
\left\|h^{1 / 2} \cdot J\left(A^{-1} p_{h} \cdot t\right)\right\|_{L^{2}(E)} \leq c_{29} \cdot\left\|A^{-1 / 2} \operatorname{Curl} \beta\right\|_{2, \omega_{E}} .
$$

Proof. Let $\psi_{E}$ denote that continuous function satisfying $0 \leq \psi_{E} \leq 1=\max \psi_{E}$ on $\omega_{E}$ and $\left.\psi_{E}\right|_{T} \in \mathcal{P}_{2}$ for each $T \in \mathcal{T}_{h}$ with $T \subset \omega_{E}$. Put $\sigma:=J\left(A^{-1} p_{h} \cdot t\right)$ which is a polynomial of degree $\leq k$ along $E$. As defined by backward and forward transformation and by continuous extension on the reference element in [V2, V3], there exists an extension operator $P: \mathcal{C}(E) \rightarrow \mathcal{C}\left(\omega_{E}\right)$ satisfying $\left.P \sigma\right|_{E}=\sigma$ and

$$
c_{30} \cdot h_{E}^{1 / 2} \cdot\|\sigma\|_{2, E} \leq\left\|\psi_{E}^{1 / 2} \cdot P \sigma\right\|_{2, \omega_{E}} \leq c_{31} \cdot h_{E}^{1 / 2} \cdot\|\sigma\|_{2, E}
$$

Similar to (6.2) (again established in [V1, V2, V3]) we gain

$$
c_{32} \cdot\|\sigma\|_{2, E}^{2} \leq\left\|\psi_{E}^{1 / 2} \cdot \sigma\right\|_{2, E}^{2}=-\int_{E}\left(\psi_{E} \cdot P \sigma\right) \cdot J\left(A^{-1} \epsilon \cdot t\right) d s .
$$

An application of (5.7) to each element $T \subset \omega_{E}$ and of (5.3) result in

$$
\begin{aligned}
& -\int_{E}\left(\psi_{E} \cdot P \sigma\right) \cdot J\left(A^{-1} \epsilon \cdot t\right) d s \\
& =-\int_{\omega_{E}}\left(A^{-1} \epsilon\right) \cdot \operatorname{Curl}\left(\psi_{E} \cdot P \sigma\right) d x-\int_{\omega_{E}}\left(\psi_{E} \cdot P \sigma\right) \cdot \operatorname{curl}\left(A^{-1} \epsilon\right) d x \\
& =\int_{\omega_{E}}\left(A^{-1} \operatorname{Curl} \beta\right) \cdot \operatorname{Curl}\left(\psi_{E} \cdot P \sigma\right) d x+\int_{\omega_{E}}\left(\psi_{E} \cdot P \sigma\right) \cdot \operatorname{curl}\left(A^{-1} p_{h}\right) d x \\
& \leq\left\|A^{-1} \operatorname{Curl} \beta\right\|_{2, \omega_{E}} \cdot\left|\psi_{E} \cdot P \sigma\right|_{1,2, \omega_{E}}+\left\|\psi_{E} \cdot P \sigma\right\|_{2, \omega_{E}} \cdot\left\|\operatorname{curl}\left(A^{-1} p_{h}\right)\right\|_{2, \omega_{E}} .
\end{aligned}
$$

Using (6.1) and (6.6) we infer

$$
\begin{gathered}
-\int_{E}\left(\psi_{E} \cdot P \sigma\right) \cdot J\left(A^{-1} \epsilon \cdot t\right) d s \leq\left\|A^{-1} \operatorname{Curl} \beta\right\|_{2, \omega_{E}} \cdot\left|\psi_{E} \cdot P \sigma\right|_{1,2, \omega_{E}} \\
+c_{26} \cdot c_{31} \cdot h_{E}^{-1 / 2} \cdot\|\sigma\|_{2, E} \cdot\left\|A^{-1 / 2} \operatorname{Curl} \beta\right\|_{2, \omega_{E}} \cdot
\end{gathered}
$$

Since $\psi_{E} \cdot P \sigma$ is a certain extension of a polynomial it follows as an inverse inequality

$$
\left|\psi_{E} \cdot P \sigma\right|_{1,2, \omega_{E}} \leq c_{33} \cdot h_{E}^{-1} \cdot\left\|\psi_{E} \cdot P \sigma\right\|_{2, \omega_{E}}
$$


(see, e.g., [V2, p.76] or [V3, Eq.(5.6)]). With (6.6), this proves

$$
\left|\psi_{E} \cdot P \sigma\right|_{1,2, \omega_{E}} \leq c_{31} \cdot c_{33} \cdot h_{E}^{-1 / 2} \cdot\|\sigma\|_{2, E} .
$$

After this, the lemma follows from (6.7)-(6.9).

Lemma 6.3. For each $T \in \mathcal{T}_{h}$,

$$
h_{T} \cdot\left\|A^{-1} p_{h}-\nabla_{h} u_{h}\right\|_{2, T} \leq c_{34} \cdot\left(\|e\|_{2, T}+h_{T} \cdot\left\|A^{-1 / 2} \epsilon\right\|_{2, T}\right) .
$$

Proof. As in (6.2), there holds

$$
c_{35} \cdot\left\|A^{-1} p_{h}-\nabla u_{h}\right\|_{2, T}^{2} \leq\left\|\psi_{T}^{1 / 2} \cdot\left(A^{-1} p_{h}-\nabla u_{h}\right)\right\|_{2, T}^{2}
$$

and integration by parts gives

$$
\begin{aligned}
& \left\|\psi_{T}^{1 / 2} \cdot\left(A^{-1} p_{h}-\nabla u_{h}\right)\right\|_{2, T}^{2} \\
& =-\int_{T} \psi_{T} \cdot A^{-1} \epsilon \cdot\left(A^{-1} p_{h}-\nabla u_{h}\right) d x-\int_{T} e \cdot \operatorname{div}\left(\psi_{T} \cdot\left(A^{-1} p_{h}-\nabla u_{h}\right)\right) d x \\
& \leq\left\|A^{-1} \epsilon\right\|_{2, T} \cdot\left\|\psi_{T} \cdot\left(A^{-1} p_{h}-\nabla u_{h}\right)\right\|_{2, T}+\|e\|_{2, T} \cdot\left|\psi_{T} \cdot\left(A^{-1} p_{h}-\nabla u_{h}\right)\right|_{1,2, T} .
\end{aligned}
$$

As in (6.4), we deduce

$$
\left|\psi_{T} \cdot\left(A^{-1} p_{h}-\nabla u_{h}\right)\right|_{1,2, T} \leq c_{36} \cdot h_{T}^{-1} \cdot\left\|\psi_{T} \cdot\left(A^{-1} p_{h}-\nabla u_{h}\right)\right\|_{2, T}
$$

and, finally, conclude the lemma from (6.11)-(6.13).

The lower bound in Theorem 2.2 is a direct consequence of Lemmas 6.1-6.3 and the (global) error decomposition (5.4).

\section{REFERENCES}

[BV] D. Braess, R. Verfürth: A posteriori error estimators for the Raviart-Thomas element. Preprint 175/1994 Fakultät für Mathematik der Ruhr-Universität Bochum.

[BF] F. Brezzi, M. Fortin: Mixed and hybrid finite element methods. Springer-Verlag 1991. MR 92d:65187

[C] P.G. Ciarlet: The finite element method for elliptic problems. North-Holland, Amsterdam 1978. MR 58:25001

[Cl] P. Clement: Approximation by finite element functions using local regularization. RAIRO Sér. Rouge Anal. Numér. R-2 77-84 (1975). MR 53:4569

[EEHJ] K. Eriksson, D. Estep, P. Hansbo, C. Johnson: Introduction to adaptive methods for differential equations. Acta Numerica (1995) 105-158. CMP 96:01

[G] P. Grisvard: Elliptic problems in nonsmooth domains. Pitman 1985. MR 86m:35044

$[\mathrm{H}]$ L. Hörmander: Linear partial differential operators. Berlin-Heidelberg-New York: Springer 1963. MR 28:4221

[LM] J.L Lions, E. Magenes: Non-homogeneous boundary value problems and applications, Vol. I. Berlin-Heidelberg-New York: Springer 1972. MR 50:2670

[N] S. Nicaise: Polygonal interface problems. Peter Lang, Frankfurt am Main, 1993. MR 94g:35067

[V1] R. Verfürth: A review of a posteriori error estimation and adaptive mesh-refinement techniques. Teubner Skripten zur Numerik. B.G. Teubner Stuttgart 1996.

[V2] R. Verfürth: A posteriori error estimates and adaptive mesh-refinement techniques. J. Comput. Appl. Math. 50 (1994) 67-83. MR 95c:65171

[V3] R. Verfürth: A posteriori error estimates for nonlinear problems. Finite element discretizations of elliptic equations. Math. Comp. 62 (1994) 445-475. MR 94j:65136

Mathematisches Seminar, Christian-Albrechts-Universität Zu Kiel, Ludewig-MeynStr. 4, D-24098 Kiel, Germany

E-mail address: cc@numerik.uni-kiel.de 